\title{
Prevalence, Predictors, and Outcomes of Prolonged Mechanical Ventilation After Endovascular Stroke Therapy
}

\author{
Hamidreza Saber ${ }^{*} \mathbb{E}$, Mohan Palla², Shaghayegh Kazemlou ${ }^{3}$, Babak B. Navi ${ }^{4}$, Albert J. Yoo ${ }^{5}$, \\ Claus Ziegler Simonsen ${ }^{6}$, Aubin Sandio ${ }^{2}$, Gary Rajahㄱ, Kasra Khatibi ${ }^{1}$, David S. Liebeskind ${ }^{1}$ and Sunil A. Sheth ${ }^{8}$
}

@ 2020 Springer Science+Business Media, LLC, part of Springer Nature and Neurocritical Care Society

\begin{abstract}
Background: To investigate the rates, predictors, and outcomes of prolonged mechanical ventilation ( $\geq 96 \mathrm{~h})$ following endovascular treatment (EVT) of ischemic stroke.

Methods: Hospitalizations with acute ischemic stroke and EVT were identified using validated codes in the National Inpatient Sample (2010-2015). The primary outcome was prolonged mechanical ventilation defined as ventilation $\geq 96$ consecutive hours. We compared hospitalizations involving prolonged ventilation following EVT with those that did not involve prolonged ventilation. Propensity score matching was used to adjust for differences between groups. Clinical predictors of prolonged ventilation were assessed using multivariable conditional logistic regression analyses.
\end{abstract}

Results: Among the 34,184 hospitalizations with EVT, 5087 (14.9\%) required prolonged mechanical ventilation. There was a decline in overall intubation and prolonged ventilation during the study period. On multivariable analysis, history of heart failure [OR $1.28(95 \% \mathrm{Cl} 1.05-1.57)$ ] and diabetes [OR $1.22(95 \% \mathrm{Cl} 1-1.50)$ ] was independent predictors of prolonged ventilation following EVT. In a sensitivity analysis of anterior circulation stroke only, heart failure [OR 1.3 (95\% Cl 1.10-1.61)], diabetes [OR 1.25 (95\% Cl 1.01-1.57)], and chronic lung disease [OR 1.31 (95\% Cl 1.03-1.66)] were independent predictors of prolonged ventilation. The weighted proportions of in-hospital mortality, post-procedural shock, acute renal failure, and intracerebral hemorrhage were higher in the prolonged ventilation group.

Conclusions: Among a nationally representative sample of hospitalizations, nearly one-in-six patients had prolonged mechanical ventilation after EVT. Heart failure and diabetes were significantly associated with prolonged mechanical ventilation following EVT. Prolonged ventilation was associated with significant increase in in-hospital mortality and morbidity.

Keywords: Ischemic stroke, Endovascular therapy, Mechanical ventilation, Epidemiology

\section{Introduction}

Despite advances in endovascular treatment (EVT) for large vessel occlusions, a considerable number of patients

\footnotetext{
*Correspondence: Hsaber@mednet.ucla.edu

${ }^{1}$ Department of Radiological Sciences, University of California Los Angeles, 635 Charles E Young Drive South, Suit 225, Los Angeles, CA 90095-7334, USA

Full list of author information is available at the end of the article
}

will not achieve favorable outcome following therapy [1]. Mechanical ventilation is frequently utilized in emergent large vessel occlusion stroke patients as part of the EVT protocol for procedural safety, or following respiratory failure or for airway protection [2]. Prolonged mechanical ventilation $(\mathrm{PMV})$ requirement $(\geq 96 \mathrm{~h})$ is an important condition following ischemic stroke and may arise due to underlying clinical comorbidities, ischemic injury impact on the brain, therapeutic interventions, or a combination 
of these factors. PMV is associated with poor in-hospital and long-term outcomes, as well as a financial burden on payers (e.g., insurers and hospitals) [2,3].

Thus, better understanding of potentially modifiable clinical risk factors for development of PMV could play an important role in the management of these patients. Yet, no prior studies have investigated the rates, predictors, and outcomes of PMV in patients undergoing EVT. Given this important gap in knowledge, we aimed to characterize the prevalence of PMV, as well as the clinical predictors and in-hospital outcomes of PMV in patients undergoing EVT using real-world data.

\section{Methods}

\section{Study Population}

The study data were obtained from the largest United States all-payer inpatient claims-based database, the National (Nationwide) Inpatient Sample (NIS). Hospitalizations for ischemic stroke involving treatment with EVT from 2010 to the third quarter of 2015 (when ICD-10 was adopted in the US) were identified using validated International Classification of Diseases-9th Revision-Clinical Modification (ICD-9-CM) diagnosis and procedural codes [4]. Patients aged less than 18 years were excluded. We excluded patients who died within the first $96 \mathrm{~h}$ of hospitalization to reduce differential misclassification. Data were checked for distribution, outliers, and missing values. Analyses of deidentified and publicly available data did not warrant an institutional review of this study.

\section{Outcomes}

The primary outcome was the development of prolonged continuous mechanical ventilation. PMV was identified using the ICD-9 code 96.72, defined as continuous invasive mechanical ventilation for $\geq 96$ consecutive hours [5-7]. Independent clinical predictors of PMV were investigated using a propensity score matched analysis. In-hospital outcomes including in-hospital mortality, intracranial hemorrhage, septic or cardiogenic shock, acute renal failure (ARF), myocardial infarction, postprocedure hemorrhage requiring transfusion, vascular complications, and length of stay (LOS) were also examined in the two groups of patients with or without PMV. Detailed information on methodology for identification of clinical conditions and outcomes using the ICD-9 codes is presented in the Online Supplement.

\section{Missing Data}

We followed the Healthcare Cost and Utilization Project (HCUP) recommendations for dealing with missing data in the NIS [8]. In NIS, race is missing approximately at $6 \%$ and the missingness for the rest of variables is less than $3 \%$. Multivariable multiple imputation was performed using the fully conditional specification (FCS) approach. The FCS method can impute missing data for different data types such as continuous, nominal, ordinal, count, and semi-continuous variables using sequential regression algorithm in IVEware [9]. Multiple imputation with 10 iterations was performed using linear regression and predictive mean matching for continuous variables, and discriminant function and logistic regression for binary or categorical variables. All the variables (demographic, sampling/hospital, clinical, and outcome) including the dependent variable were included in the modeling of multiple imputation to preserve the variance [10].

\section{Propensity Score Matching}

Propensity score matching was used to adjust for differences between the two groups. Propensity scores were estimated using a logistic regression model (Table 1). We performed the Variable or Full matching method using the optimal matching algorithm and applied a caliper radius of 0.1 times the standard deviation of the estimated propensity score. We performed a 1:3 case/control matching and evaluated the balance between two groups both before and after propensity score matching

\begin{tabular}{|c|c|c|c|}
\hline \multirow[t]{3}{*}{ Characteristic } & \multicolumn{3}{|c|}{ Before matching } \\
\hline & \multicolumn{2}{|c|}{$\begin{array}{l}\text { Prolonged mechanical } \\
\text { ventilation }\end{array}$} & \multirow[t]{2}{*}{$P$ value } \\
\hline & Yes & No & \\
\hline Weighted observation & $5087(14.9)$ & $29,097(85.1)$ & \\
\hline Age (mean, SD) & $64.3( \pm 0.46)$ & $66.4( \pm 0.20)$ & 0.001 \\
\hline Female & $2275(44.7)$ & $14,978(51.5)$ & 0.001 \\
\hline \multicolumn{4}{|l|}{ Race } \\
\hline White, $n(\%)$ & $3082(60.6)$ & $19,644(67.5)$ & 0.003 \\
\hline Black, $n(\%)$ & $819(16.1)$ & $3581(12.3)$ & 0.014 \\
\hline Hispanic, $n(\%)$ & $500(9.8)$ & $2346(8.1)$ & 0.160 \\
\hline Asian or Pacific, $n(\%)$ & $277(5.4)$ & $1626(5.6)$ & 0.900 \\
\hline Obesity, $n(\%)$ & $694(13.6)$ & $3151(10.9)$ & 0.010 \\
\hline Hypertension, $n(\%)$ & $3697(72.7)$ & $21,421(73.7)$ & 0.530 \\
\hline Diabetes, $n(\%)$ & $1277(25.1)$ & $6096(20.9)$ & 0.001 \\
\hline CAD, $n(\%)$ & $1288(25.3)$ & 7805 (26.8) & 0.310 \\
\hline Heart failure, $n(\%)$ & $1237(24.3)$ & $5518(18.9)$ & $<0.001$ \\
\hline Chronic lung disease, $n(\%)$ & $888(17.4)$ & $4101(14.1)$ & 0.008 \\
\hline $\begin{array}{l}\text { Pulmonary circulation disorders, } \\
n(\%)\end{array}$ & $438(8.6)$ & $1753(6.1)$ & 0.006 \\
\hline Renal failure, $n(\%)$ & $530(10.4)$ & $2782(9.5)$ & 0.406 \\
\hline Peripheral arterial disease, $n(\%)$ & $886(17.4)$ & $3565(12.2)$ & 0.001 \\
\hline
\end{tabular}

Values are mean $\pm S D$ for continuous variables and percent for categorical variables. CAD: Coronary artery disease 
by comparing the standardized difference of means (Table 1) and distribution of covariates used in the propensity score modeling.

\section{Statistical Analysis}

All the analyses (univariable and multivariable) were performed using weighted data using survey-specific design (strata, clusters, and discharge weights). We analyzed 10 imputed datasets separately and combined the results using Rubin's rules to produce key estimates and standard error [10]. Categorical variables were expressed as sum of weights (weighted proportion) and continuous variables as weighted mean ( \pm standard error). We performed multivariable conditional logistic regression models of matched observations to identify the independent predictors of PMV following EVT. Statistically significant baseline clinical covariates in univariable analysis with $P$ value of $<0.05$ were used in modeling conditional logistic regression. Results of regression models were reported using odds ratios and $95 \%$ confidence intervals. Statistical analysis was performed using SAS Version 9.4 (SAS Institute Inc., Cary, North Carolina) and IVEware Version 0.3.

\section{Sensitivity Analysis}

We performed a sensitivity analysis restricting the cohort to patients who had claims for anterior circulation LVOs only; to identify these patients, we used a previously published algorithm of ICD-9-CM diagnostic codes to exclude strokes involving the basilar or vertebral arteries (433.01 and 433.2) [11]. Also, a separate sensitivity analysis was performed restricted to patients receiving EVT and concomitant intravenous tissue-type plasminogen activator (tPA) administration (99.10 ICD-9 code) [4].

\section{Data Availability}

Data used in this study are publicly available via HCUP. Requests for access to the data are possible upon reasonable request.

\section{Results}

\section{Baseline Characteristics}

Nationally, there was an estimated total of 34,184 EVT hospitalizations between January 1, 2010 and September 30, 2015, and the estimated number of adults who required PMV following EVT in this period was 5087 (14.9\%). The rates of PMV requirement decreased from $19.4 \%$ in 2010 to $13.1 \%$ in 2015 (Fig. 1). We observed a continuous decline in the rate of overall intubation (with short-term or prolonged ventilation requirement) during hospitalizations for EVT during the study period (Fig. 1).

Table 1 shows the baseline characteristics for clinical and hospital level factors before matching. Significant differences were seen in demographics and sampling/

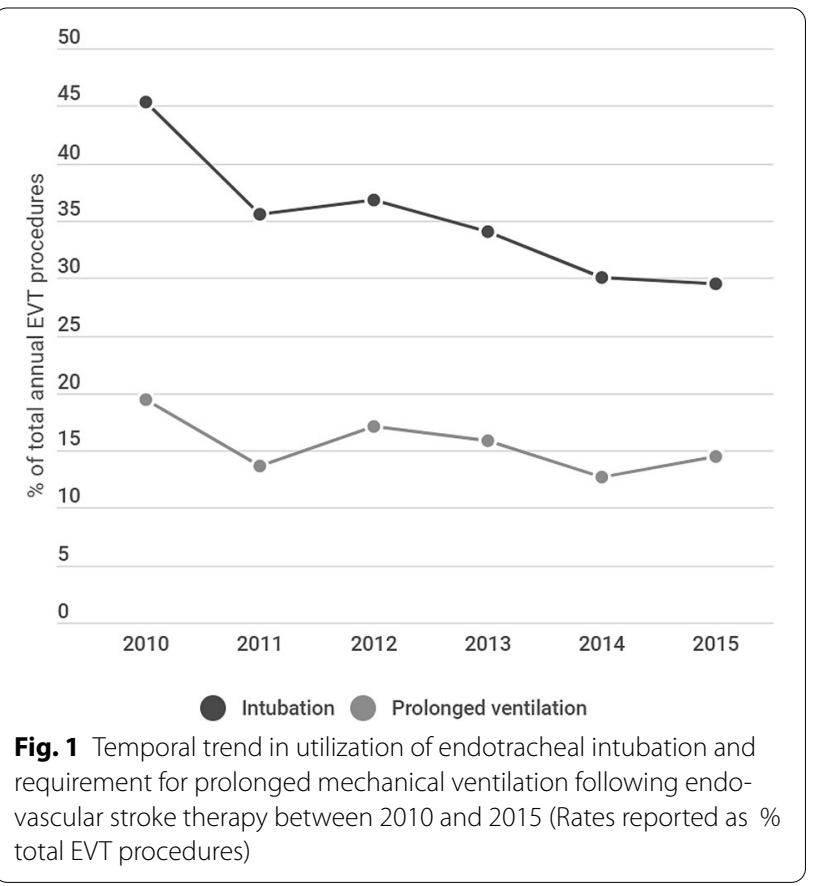

hospital characteristics (Table 2). After adjustment with the use of Variable or Full matching, all these characteristics were well balanced (Table 2). The clinical covariates of patients, with and without PMV, following EVT after propensity score matching are shown in Table 3.

\section{Predictors of Prolonged Mechanical Ventilation Following EVT}

After propensity score matching, heart failure and diabetes were the only covariates that were significantly associated with PMV in univariable analysis (Table 3). In multivariable analysis, history of heart failure [OR 1.28 (95\% CI 1.05-1.57)] and diabetes [OR 1.22 (95\% CI $1-1.5)]$ remained as independent predictors of PMV following EVT.

\section{Secondary in-Hospital Outcomes}

In-hospital mortality [1325 (32.1\%) vs. 892 (8.2\%); $P<0.001]$ was significantly higher among patients who required PMV following EVT. The weighted proportions of post-procedural shock, acute renal failure, and intracerebral hemorrhage were significantly higher in the PMV group and the trends were similar to the weighted proportions for in-hospital mortality (Table 4). No significant difference was seen between the two groups for the outcomes of acute MI, vascular complications, or postprocedure hemorrhage requiring transfusion (Table 4). 
Table 2 Standardized mean differences for hospital- and patient-level variables before and after matching

\begin{tabular}{|c|c|c|}
\hline \multirow[t]{2}{*}{ Propensity score model variables } & \multicolumn{2}{|c|}{ Standardized difference of means } \\
\hline & Before matching & $\begin{array}{l}\text { Propensity score } \\
\text { matched data }\end{array}$ \\
\hline Age & 0.140 & 0.076 \\
\hline Female & 0.130 & 0.030 \\
\hline Race & 0.200 & 0.114 \\
\hline Elective versus non-elective admission & 0.01 & 0.009 \\
\hline Admission day is a weekend & 0.050 & 0.016 \\
\hline Primary expected payer & 0.195 & 0.105 \\
\hline Number of chronic conditions & 0.191 & 0.052 \\
\hline Number of diagnoses & 0.750 & 0.156 \\
\hline Number of procedures & 1.23 & 0.334 \\
\hline NIS discharge weight & 0.110 & 0.025 \\
\hline NIS hospital stratum & 0.560 & 0.478 \\
\hline Region of hospital & 0.110 & 0.067 \\
\hline Number of universe discharges in the stratum & 0.100 & 0.001 \\
\hline Number of universe hospitals in the stratum & 0.025 & 0.007 \\
\hline Number of sample discharges in the stratum & 0.080 & 0.005 \\
\hline Number of sample hospitals in the stratum & 0.050 & 0.030 \\
\hline Total number of discharges from hospital & 0.100 & 0.028 \\
\hline
\end{tabular}

NIS National inpatient sample

Table 3 Clinical characteristics in the cohort following 3:1 propensity score matching

\begin{tabular}{|c|c|c|c|}
\hline \multirow[t]{3}{*}{ Characteristic } & \multicolumn{3}{|c|}{ Propensity score matched data } \\
\hline & \multicolumn{2}{|c|}{$\begin{array}{l}\text { Prolonged mechanical } \\
\text { ventilation }\end{array}$} & \multirow[t]{2}{*}{$P$ value } \\
\hline & Yes & No & \\
\hline Unweighted observation, $n$ (\%) & $808(26.9)$ & $2193(73.1)$ & \\
\hline Weighted observation, n (\%) & 4008 (26.9) & $10,881(73.1)$ & \\
\hline Age (mean, SD) & $65.2( \pm 0.54)$ & $66( \pm 0.34)$ & 0.180 \\
\hline Female, $n(\%)$ & $1824(45.5)$ & $4957(45.5)$ & 0.980 \\
\hline \multicolumn{4}{|l|}{ Race } \\
\hline White, $n(\%)$ & $2501(62.4)$ & $6978(64.1)$ & 0.430 \\
\hline Black, $n(\%)$ & $608(15.2)$ & $1606(14.7)$ & 0.800 \\
\hline Hispanic, $n(\%)$ & $361(9.1)$ & $883(8.1)$ & 0.480 \\
\hline Asian or Pacific, $n(\%)$ & $225(5.6)$ & $562(5.1)$ & 0.660 \\
\hline Obesity, $n(\%)$ & $501(12.5)$ & $1355(12.4)$ & 0.900 \\
\hline Hypertension, n (\%) & $2913(72.6)$ & $7890(72.5)$ & 0.930 \\
\hline Diabetes, $n(\%)$ & $996(24.8)$ & $2286(21.1)$ & 0.040 \\
\hline$C A D, n(\%)$ & $996(24.8)$ & $3035(28)$ & 0.113 \\
\hline Heart failure, $n(\%)$ & $1002(24.9)$ & $2220(20.4)$ & 0.010 \\
\hline Chronic lung disease, $n$ (\%) & $714(17.8)$ & $1590(14.6)$ & 0.066 \\
\hline $\begin{array}{l}\text { Pulmonary circulation disorders, } \\
n(\%)\end{array}$ & $330(8.3)$ & $678(6.2)$ & 0.070 \\
\hline Renal failure, $n(\%)$ & $413(10.3)$ & $1187(10.8)$ & 0.680 \\
\hline Peripheral arterial disease, $n(\%)$ & $666(16.6)$ & $1575(14.5)$ & 0.170 \\
\hline
\end{tabular}

Values are mean \pm SD for continuous variables and percent for categorical variables. $C A D$ coronary artery disease

\section{Sensitivity Analysis}

Table 5 demonstrates the baseline characteristics of the propensity score matched analysis cohort restricted to strokes of the anterior circulation. In this cohort, history of heart failure [OR 1.30 (95\% CI 1.10-1.61), $P=0.01$ ], diabetes [OR 1.25 (95\% CI 1.01-1.57, $P=0.04)$ ], and chronic lung disease [OR 1.31 (95\% CI 1.03-1.66), $P=0.03]$ was significantly associated with higher risk of PMV. In a separate analysis restricted to patients treated with $\mathrm{TPA}$ in addition to EVT, history of heart failure [OR 1.34 (95\% CI 1.00-1.80), $P=0.05$ ] was the only independent predictor of PMV.

\section{Discussion}

In this analysis of nationwide discharge data on stroke patients undergoing EVT, about $15 \%$ of patients developed PMV. A history of CHF and diabetes independently predicted for PMV; and PMV was significantly associated with higher risks of mortality, acute kidney injury, and prolonged hospitalization. We observed a decline in utilization of mechanical ventilation during the study period.

To our knowledge, this is the first study that explored the rates, predictors, and outcomes of PMV in patients undergoing EVT. Prior studies have mainly examined long-term outcomes and predictors of functional recovery in the patient population requiring prolonged ventilation after stroke. In one study, poor outcome, defined as Glasgow Outcome Scale score 1-3, occurred in $74 \%$ of 
Table 4 In-hospital outcomes in patient requiring prolonged ventilation in NIS (2010-2015)

\begin{tabular}{|c|c|c|c|}
\hline \multirow[t]{3}{*}{ Outcomes } & \multicolumn{3}{|c|}{ Propensity score matched data } \\
\hline & \multicolumn{2}{|c|}{ Prolonged mechanical ventilation } & \multirow[t]{2}{*}{$P$ value } \\
\hline & Yes & No & \\
\hline Inpatient mortality, n (\%) & $1325(32.1)$ & $892(8.2)$ & $<0.001$ \\
\hline Postoperative shock, $n$ (\%) & $327(8.1)$ & $274(2.5)$ & $<0.001$ \\
\hline Postoperative cardiogenic shock, $n(\%)$ & $79(1.9)$ & $81(0.7)$ & 0.041 \\
\hline Postoperative septic shock, n (\%) & $196(4.9)$ & $143(1.3)$ & $<0.001$ \\
\hline Acute kidney Injury, n (\%) & $918(22.9)$ & $1512(13.9)$ & $<0.001$ \\
\hline Acute $\mathrm{MI}, n(\%)$ & $312(7.8)$ & $594(5.4)$ & 0.064 \\
\hline Acute intracranial hemorrhage, $n(\%)$ & $1160(28.9)$ & $2524(23.2)$ & 0.003 \\
\hline Length of stay & $17.93( \pm 0.49)$ & $11.55( \pm 0.24)$ & $<0.001$ \\
\hline Postoperative hemorrhage, $n(\%)$ & $41(1.1)$ & $73(0.7)$ & 0.420 \\
\hline Vascular complications, n (\%) & $137(3.4)$ & $417(3.8)$ & 0.612 \\
\hline
\end{tabular}

MI myocardial infarction, NIS National inpatient sample

Table 5 Sensitivity analysis (Anterior circulation cohort only)

\begin{tabular}{|c|c|c|c|}
\hline & \multicolumn{3}{|c|}{ Propensity score matched data } \\
\hline & \multicolumn{2}{|c|}{$\begin{array}{l}\text { Prolonged mechanical } \\
\text { ventilation }\end{array}$} & \multirow[t]{2}{*}{$P$ value } \\
\hline & Yes & No & \\
\hline Age (mean, SD) & $65.36( \pm 0.5)$ & $66.18(0.4)$ & 0.250 \\
\hline Female, $n(\%)$ & $1652(46.7)$ & $4542(46.9)$ & 0.880 \\
\hline \multicolumn{4}{|l|}{ Race } \\
\hline White, $n(\%)$ & $2229(62.9)$ & $6193(64.9)$ & 0.620 \\
\hline Black, $n(\%)$ & $543(15.3)$ & $1442(14.9)$ & 0.810 \\
\hline Hispanic, n (\%) & $333(9.4)$ & $834(8.6)$ & 0.580 \\
\hline Asian or Pacific, $n(\%)$ & $200(5.6)$ & $549(5.6)$ & 0.960 \\
\hline Hypertension, $n(\%)$ & $2572(72.6)$ & $6951(71.9)$ & 0.760 \\
\hline Diabetes, $n(\%)$ & $883(24.9)$ & $1986(20.5)$ & 0.029 \\
\hline$C A D, n(\%)$ & $875(24.7)$ & $2672(27.6)$ & 0.168 \\
\hline Heart failure, $n(\%)$ & $929(26.2)$ & $2015(20.8)$ & 0.006 \\
\hline Chronic lung disease, $n$ (\%) & $670(18.1)$ & $1427(14.7)$ & 0.017 \\
\hline $\begin{array}{l}\text { Pulmonary circulation disorders, } \\
n(\%)\end{array}$ & $275(7.8)$ & $626(6.5)$ & 0.301 \\
\hline Renal failure, $n(\%)$ & $374(10.5)$ & $1052(10.8)$ & 0.857 \\
\hline Peripheral arterial disease, $n$ (\%) & $594(16.7)$ & $1385(14.3)$ & 0.150 \\
\hline
\end{tabular}

$C A D$ coronary artery disease

patients at 1 year in those requiring tracheostomy [12]. In another study of 74 patients with ischemic stroke, mortality rate was $55 \%$ in those who required mechanical ventilation [13]. Signs of brainstem dysfunction and male gender predicted mortality in patients requiring ventilation, whereas stroke location did not influence long-term outcomes. In another study of 124 consecutive stroke patients who required mechanical ventilation, $52 \%$ died in the neurological intensive care unit [2]. In this study, Glasgow Coma Scale score $<10$ and intubation due to coma/acute respiratory failure were independent predictors of death at 2 months [2]. In a study using NIS data, pneumonia was found to be a significant predictor of tracheostomy [14]. However, this study was not able to distinguish between the timing of pneumonia relative to the timing of tracheostomy. Ventilator associated pneumonia is a common complication of mechanical ventilation with greatest risk occurring during the first 5 days of mechanical ventilation [15]. Thus, pneumonia may more likely represent an in-hospital infection prior to tracheostomy rather than a baseline clinical risk indicator of prolonged ventilation. Unlike these prior studies, we considered PMV as an important stand-alone clinical outcome and explored predictors of PMV using baseline clinical characteristics.

Possible explanations for the observed associations include worsening of pulmonary edema and alteration of physiologic homeostasis due to cardiac dysfunction and poor myocardial contractile reserve, particularly in the setting of stroke-related systemic inflammatory response. This process could lead to an increased capillary permeability including pulmonary circulation which may in turn contribute to respiratory failure and PMV in those with poor cardiac function. Furthermore, patients with heart failure and diabetes have other associated comorbidities which contribute to a greater systemic burden of large vessel occlusive stroke. Cabello and colleagues identified heart failure as the cause of $42 \%$ of failures of spontaneous breathing trials in a large cohort of medical ICU patients [16]. In an externally validated risk score by Sharma et al., low left ventricular ejection fraction was reported as an independent predictor of intubation 
following cardiac surgery [17]. Interestingly, in a study of 117 patients with heart failure and 634 patients without, the authors reported that patients with PMV and heart failure had significantly lower weaning rate and higher mortality rate compared with PMV patients without heart failure. Also, a randomized clinical trial showed a BNP-driven fluid management protocol that decreased the duration of mechanical ventilation, especially in patients with chronic heart failure [18]. Diabetes is an important predictor of stroke severity at onset, as well as radiographic stroke progression at 24-h after stroke likely reflecting the fact that diabetes at baseline is associated with impaired collateral flow [19]. Therefore, a higher likelihood of requirement for PMV may represent poor collateral circulation and fast core infarct progression in this group of patients. Diabetes has also been shown to be associated with a higher likelihood of respiratory or bloodstream infections in ICU patients which may have contributed to PMV requirement after endovascular stroke therapy [20]. Because of the lack of important neurological clinical information associated with PMV, including stroke severity, exact location and size of stroke, etc., the results of the article may not completely reflect the risk factors of PMV.

We observed a decline in rates of intubation utilization (overall general anesthesia) and PMV over time during the period of this analysis. However, our analysis of claims data for PMV is unable to differentiate whether patients were initially intubated for elective reasons prior to the endovascular procedure, or for medical comorbidities and complications requiring intubation and mechanical ventilation. Results from the general or local anesthesia in intra-arterial therapy (GOLIATH) trial showed no significant difference in infarct growth, clinical outcomes [21], or ventilator associated pneumonia [22] between patients randomized to the general anesthesia and those randomized to the conscious sedation. Thus, prolonged ventilation requirement is more likely associated with the underlying clinical comorbidities and the acute ischemic sequala, rather than the elective choice of pre-procedural general anesthesia. Taken together, the observed decline in rates of prolonged ventilation requirement over time would more likely represent recent improvements in EVT and post-interventional clinical care, rather than merely a decrease in rates of general anesthesia utilization prior to EVT. In addition, practice changes in the field could have implications on our findings via patient selection and systems of care, as prior to the landmark endovascular stroke trials, only the most severe LVOs were more likely to be selected for EVT and these patients may have had prolonged intubation as the result of their underlying stroke severity.
We believe that the findings of this analysis have important implications because it demonstrates the important potentially modifiable risk factors for PMV following EVT. A proper management plan such as better treatment optimization of patients with CHF or diabetes before and after EVT could help minimize the morbidity and mortality in patients with large vessel occlusive strokes. Our results also may have implications for future research on predictors of respiratory complications following EVT as it supports the investigation of more sensitive biomarkers for cardiopulmonary failure, such as BNP.

Our analysis cannot characterize whether diabetes or heart failure equally relates to the risk of PMV in patients with elective vs non-elective (e.g., respiratory failure) intubation prior to EVT. Therefore, future clinical studies will be needed to examine the risk of prolonged ventilation in different subgroups of ischemic stroke patients that undergo intubation prior to endovascular stroke therapy. In fact, the choice of anesthetic strategy (elective intubation vs conscious sedation) on functional outcome is an unresolved matter, whereas a recent individual patient meta-analysis of $368 \mathrm{EVT}$ who were randomized to general anesthesia or procedural sedation showed better functional outcomes in those who received general anesthesia [23], other retrospective studies reported worse functional outcome in EVT patients with general anesthesia [24, 25]. Therefore, future studies need to examine whether underlying comorbidities such as diabetes or heart failure would interact with the association between elective general anesthesia prior to EVT and functional outcomes.

Our investigation has several strengths. It represents a comprehensive examination of a large, real-world patient population with endovascular stroke therapy. While missing values are typically excluded in many of the analyses of the nationwide data, we used a robust methodology to impute missing values following guidelines provided by the HCUP. We also utilized a robust matching algorithm, i.e., the Full/Variable propensity score matching, that allows optimal adjustment for differences between the two groups (with or without prolonged ventilation requirement) using all available variables in the database.

Our study has limitations. Despite efforts to minimize bias, including a robust matching strategy to adjust for baseline differences between groups and a sensitivity analysis restricted to a more homogenous group of patients, residual confounding by measured or unmeasured variables remain possible. The NIS lacks clinical information regarding stroke severity, exact location, and size of stroke; the time from symptom onset to treatment; EVT recanalization rates; why patients were 
initially intubated and the status of advanced directive for maximal vs minimal life support. Therefore, we cannot pinpoint whether our findings are differentially mediated by these factors. However, we performed two sets of sensitivity analyses restricted to patients with slightly different clinical characteristics (IV tPA eligible vs not eligible; anterior circulation vs vertebrobasilar strokes) which yielded similar results. Additionally, this analysis spanned a time of substantial practice changes that could influence the observed results. In this analysis, we used a small and precise caliper $(0.1 \mathrm{SD})$ for matching that increases the quality of the matches that are made; however, the trade-off is that the number of unmatched cases may increase, that may increase the likelihood of bias due to incomplete matching. Finally, the identification of diagnoses and procedures was largely dependent on ICD-9-CM codes collected for billing purposes and thus may be susceptible to measurement error. However, this is more likely to be nondifferential in nature and therefore would be less likely to alter the observed associations.

\section{Conclusion}

In summary, heart failure and diabetes were independent predictors of PMV following EVT. PMV was associated with greater risk of in-hospital mortality, acute renal failure, and increased length of stay following EVT. Early identification of these potentially modifiable clinical factors and implementing appropriate management strategies, such as patient positioning and careful glycemic and fluid balance management, may help improve outcomes in patients admitted to intensive care units following EVT.

\section{Electronic supplementary material}

The online version of this article (https://doi.org/10.1007/s12028-020-01125-9) contains supplementary material, which is available to authorized users.

\begin{abstract}
Author details
${ }^{1}$ Department of Radiological Sciences, University of California Los Angeles, 635 Charles E Young Drive South, Suit 225, Los Angeles, CA 90095-7334, USA. 2 Department of Cardiology, Wayne State University School of Medicine, Detroit, MI, USA. ${ }^{3}$ Danaher Lab, San Jose, CA, USA. ${ }^{4}$ Clinical and Translational Neuroscience Unit, Department of Neurology, Feil Family Brain and Mind Research Institute, Weill Cornell Medicine, New York, NY, USA. ${ }^{5}$ Neurointervention, Texas Stroke Institute, Dallas-Fort Worth, TX, USA. ${ }^{6}$ Department of Neurology, Aarhus University Hospital, Aarhus, Denmark. ${ }^{7}$ Department of Neurosurgery, Jacobs School of Medicine and Biomedical Sciences, University of Buffalo, Buffalo, NY, USA. ${ }^{8}$ Department of Neurology, UT Health McGovern School of Medicine, Houston, TX, USA.
\end{abstract}

\section{Author Contributions}

HS and MP performed the design, statistical analysis, and interpretation of the results. $\mathrm{HS}, \mathrm{SS}, \mathrm{AS}, \mathrm{DL}$, and $\mathrm{BN}$ prepared the manuscript. SS, DL, BN, AY, CS, RJ, and GR revised the draft paper for intellectual content.

\section{Source of support}

None.

\section{Conflicts of Interest}

None

\section{Publisher's Note}

Springer Nature remains neutral with regard to jurisdictional claims in published maps and institutional affiliations.

Received: 5 April 2020 Accepted: 30 September 2020

Published online: 21 October 2020

\section{References}

1. Goyal M, Menon BK, van Zwam WH, et al. Endovascular thrombectomy after large-vessel ischaemic stroke: a meta-analysis of individual patient data from five randomised trials. Lancet. 2016;387:1723-31.

2. Steiner T, Mendoza G, De Georgia M, Schellinger P, Holle R, Hacke W. Prognosis of stroke patients requiring mechanical ventilation in a neurological critical care unit. Stroke. 1997;28:711-5.

3. Lahiri S, Mayer SA, Fink ME, et al. Mechanical ventilation for acute stroke: a multi-state population-based study. Neurocrit Care [online serial]. 2015;23:28-32.

4. Saber H, Navi BB, Grotta JC, et al. Real-world treatment trends in endovascular stroke therapy. Stroke. 2019:50:683-9.

5. Nanchal R, Kumar G, Majumdar T, et al. Utilization of mechanical ventilation for asthma exacerbations: analysis of a national database. Respir Care. 2014;59:644-53.

6. Statistical Brief \#108 [online]. Accessed at: https://www.hcupus.ahrq.gov/ reports/statbriefs/sb108.jsp. Accessed June 2, 2019

7. Zilberberg MD, Shorr AF. Prolonged acute mechanical ventilation and hospital bed utilization in 2020 in the United States: implications for budgets, plant and personnel planning. BMC Health Serv Res. 2008;8:242.

8. HCUP Methods Series Missing Data Methods for the NIS and the SID Report \# 2015-01 [online]. Accessed at: http://www.hcup-us.ahrq.gov/ reports/methods/methods.jsp. Accessed March 28, 2019.

9. Raghunathan TE, Lepkowski JM, Van Hoewyk J, Solenberger P, van Hoewyk J. A multivariate technique for multiply imputing missing values using a sequence of regression models key words: item nonresponse; missing at random; multiple imputation; nonignorable missing mechanism; regression; sampling properties and simulations [online]. Surv Methodol. 2001.

10. Marshall A, Altman DG, Holder RL, Royston P. Combining estimates of interest in prognostic modelling studies after multiple imputation: current practice and guidelines. BMC Med Res Methodol. 2009;9:57.

11. Qureshi Al, Chaudhry SA, Hassan AE, et al. Thrombolytic treatment of patients with acute ischemic stroke related to underlying arterial dissection in the United States. Arch Neurol. 2011;68:1536.

12. Rabinstein AA, Wijdicks EFM. Outcome of survivors of acute stroke who require prolonged ventilatory assistance and tracheostomy. Cerebrovasc Dis. 2004;18:325-31.

13. Gujjar AR, Deibert E, Manno EM, Duff S, Diringer MN. Mechanical ventilation for ischemic stroke and intracerebral hemorrhage: indications, timing, and outcome. Neurology. 1998;51:447-51.

14. Walcott BP, Boehm KM, Stapleton CJ, Mehta BP, Nahed BV, Ogilvy CS Retrievable stent thrombectomy in the treatment of acute ischemic stroke: analysis of a revolutionizing treatment technique. J Clin Neurosci. 2013:20:1346-9.

15. Kalanuria AA, Ziai W, Zai W, Mirski M. Ventilator-associated pneumonia in the ICU. Crit Care [online serial]. BioMed Central; 2014;18:208.

16. Cabello B, Thille AW, Roche-Campo F, Brochard L, Gómez FJ, Mancebo J. Physiological comparison of three spontaneous breathing trials in difficult-to-wean patients. Intensive Care Med. 2010:36:1171-9.

17. Sharma V, Rao V, Manlhiot C, Boruvka A, Fremes S, Wąsowicz M. A derived and validated score to predict prolonged mechanical ventilation in patients undergoing cardiac surgery. J Thorac Cardiovasc Surg. Elsevier. 2017:153:108-15.

18. Dessap AM, Roche-Campo F, Kouatchet A, et al. Natriuretic peptidedriven fluid management during ventilator weaning. Am J Respir Crit Care Med. 2012;186:1256-63. 
19. Kim J-T, Liebeskind DS, Jahan R, et al. Impact of hyperglycemia according to the collateral status on outcomes in mechanical thrombectomy. Stroke Lippincott Williams Wilkins. 2018;49:2706-14.

20. Vardakas KZ, Siempos II, Falagas ME. Diabetes mellitus as a risk factor for nosocomial pneumonia and associated mortality. Diabet Med. 2007;24:1168-71.

21. Simonsen CZ, Yoo AJ, Sørensen LH, et al. Effect of general anesthesia and conscious sedation during endovascular therapy on infarct growth and clinical outcomes in acute ischemic stroke. JAMA. 2018;75:470.

22. Sørensen LH, Speiser L, Karabegovic S, et al. Safety and quality of endovascular therapy under general anesthesia and conscious sedation are comparable: results from the GOLIATH trial. Epub: J Neurointerv Surg; 2019.
23. Schönenberger S, Hendén PL, Simonsen CZ, et al. Association of general anesthesia vs procedural sedation with functional outcome among patients with acute ischemic stroke undergoing thrombectomy. JAMA. 2019;322:1283.

24. Campbell BCV, van Zwam WH, Goyal M, et al. Effect of general anaesthesia on functional outcome in patients with anterior circulation ischaemic stroke having endovascular thrombectomy versus standard care: a metaanalysis of individual patient data. Lancet Neurol. 2018;17:47-53.

25. Berkhemer OA, van den Berg LA, Fransen PSS, et al. The effect of anesthetic management during intra-arterial therapy for acute stroke in MR CLEAN. Neurology. 2016;87:656-64. 